\title{
CORRECTION
}

\section{Publisher Correction: Molecular basis of vasohibins-mediated detyrosination and its impact on spindle function and mitosis}

Shanhui Liao', Girish Rajendraprasad $\mathbb{D}^{2}$, Na Wang ${ }^{3}$, Susana Eibes ${ }^{2}$, Jun Gao', Huijuan $\mathrm{Yu}^{1}$, Gao Wu' ${ }^{1}$ Xiaoming Tu${ }^{1}$, Hongda Huang $\mathbb{1}^{3}$, Marin Barisic $\mathbb{E}^{2,4}$ and Chao Xu (i) ${ }^{1}$

Cell Research (2019) 29:778; https://doi.org/10.1038/s41422-019-0215-y

Correction to: Cell Research https://doi.org/10.1038/s41422-0190187-y, published online 6 June 2019

In the initial published version of this article, there was an inadvertent omission from the Acknowledgements that work in the lab of MB is supported by grants from Danish Cancer Society
Scientific Committee (KBVU; R146-A9322) and the Lundbeck Foundation (R215-2015-4081). This omission does not affect the description of the results or the conclusions of this work. The publisher apologizes for any inconvenience this has caused to the readers.

${ }^{1}$ Hefei National Laboratory for Physical Sciences at Microscale and School of Life Sciences, University of Science and Technology of China, Hefei, China; ${ }^{2}$ Cell Division and Cytoskeleton, Danish Cancer Society Research Center, 2100 Copenhagen, Denmark; ${ }^{3}$ Department of Biology, Southern University of Science and Technology, Shenzhen, China and ${ }^{4}$ Department of Cellular and Molecular Medicine, Faculty of Health Sciences, University of Copenhagen, 2100 Copenhagen, Denmark

Correspondence: Hongda Huang (huanghd@sustech.edu.cn) or Marin Barisic (barisic@cancer.dk) or Chao Xu (xuchaor@ustc.edu.cn)

These authors contributed equally: Shanhui Liao, Girish Rajendraprasad, Na Wang

Published online: 8 August 2019 